\title{
Compact bifluid hybrid stars: hadronic matter mixed with self-interacting fermionic asymmetric dark matter
}

\author{
Somnath Mukhopadhyay ${ }^{1, a}$, Debasis Atta ${ }^{1,2, b}$, Kouser $\operatorname{Imam}^{1,3, c}$, D. N. Basu ${ }^{1, d}$, C. Samanta ${ }^{4, e}$ \\ ${ }^{1}$ Variable Energy Cyclotron Centre, HBNI, 1/AF Bidhan Nagar, Kolkata 700 064, India \\ ${ }^{2}$ Government General Degree College, Kharagpur II, West Bengal 721 149, India \\ ${ }^{3}$ Department of Physics, Aliah University, IIA/27, New Town, Kolkata 700156, India \\ ${ }^{4}$ Department of Physics and Astronomy, Virginia Military Institute, Lexington, VA 24450, USA
}

Received: 1 February 2017 / Accepted: 20 June 2017 / Published online: 3 July 2017

(C) The Author(s) 2017, corrected publication August 2017. This article is an open access publication

\begin{abstract}
The masses and radii of non-rotating and rotating configurations of pure hadronic stars mixed with selfinteracting fermionic asymmetric dark matter are calculated within the two-fluid formalism of stellar structure equations in general relativity. The Equation of State (EoS) of nuclear matter is obtained from the density dependent M3Y effective nucleon-nucleon interaction. We consider the dark matter particle mass of $1 \mathrm{GeV}$. The EoS of self-interacting dark matter is taken from two-body repulsive interactions of the scale of strong interactions. We explore the conditions of equal and different rotational frequencies of nuclear matter and dark matter and find that the maximum mass of differentially rotating stars with self-interacting dark matter to be $\sim 1.94 \mathrm{M}_{\odot}$ with radius $\sim 10.4 \mathrm{~km}$.
\end{abstract}

\section{Introduction}

In the universe there are large empty regions and dense regions where the galaxies are distributed. This distribution is called the cosmic web that is speculated to be governed by the action of gravity on the invisible mysterious "dark matter". Recently, a research group led by Hiroshima University has suggested that the Cancer Constellation has nine such large concentrations of dark matter, each the mass of a galaxy cluster [1].

The original version of this article was revised: on page 3 first paragraph $a_{v}$ was not correctly displayed.

\footnotetext{
a e-mail: somnathm@vecc.gov.in

b e-mail: debasisa906@gmail.com

c e-mail: kouserblackhole@gmail.com

de-mail: dnb@vecc.gov.in

e e-mail: samantac@vmi.edu
}

Various theoretical models of dark matter are widespread, ranging from cold dark matter to warm dark matter to hot dark matter and from symmetric to asymmetric dark matter [2-6]. Recent advances in cosmological precision tests further consolidate the minimal cosmological standard model, indicating that the universe contains $4.9 \%$ ordinary matter, $26.8 \%$ dark matter and $68.3 \%$ dark energy. Although being five times more abundant than ordinary matter, the basic properties of dark matter, such as particle mass and interactions are unsolved.

A dark star composed mostly of normal matter and dark matter may have existed early in the universe before conventional stars were able to form. Those stars generate heat via annihilation reactions between the dark-matter particles. This heat prevents such stars from collapsing into the relatively compact sizes of modern stars and therefore prevent nuclear fusion among the normal matter atoms from being initiated [7].

One theory is that dark matter could be made of particles called axions. Unlike protons, neutrons and electrons that make up ordinary matter, axions can share the same quantum energy state. They also attract each other gravitationally, so they clump together. Dark matter is hard to study because it does not interact much with ordinary matter but axion dark matter could theoretically be observed in the form of Bose stars [8]. The Bose-Einstein condensation may come from the bosonic features of dark matter models. A phase transition to condensation can occur either when the temperature decreases to below the critical value or when the density exceeds the critical value [9].

The neutron stars could capture weakly interacting dark matter particles (WIMPs) because of their strong gravitational field, high density and finite, but very small, WIMPto-nucleon cross section. In fact, if there is no baryon-dark matter interaction, a purely baryonic neutron star would not 
capture dark matter at all. A dark star of comparable mass may as well accrete neutron star matter to form a dark matter dominated neutron star. In 1978, Steigman et al. [10] suggested that capture of WIMPs by individual stellar objects could affect the stellar structure and evolution. The effects of self-annihilating dark matter on first-generation stars and on the evolution path of main sequence stars have been studied extensively $[11,12]$. For non self-annihilating dark matter, its impact on main sequence stars [13] and neutron stars [14,15] has been studied in different dark matter models. Gravitational effects of non self-annihilating condensate dark matter on compact stellar objects has been studied [16] assuming dark matter as ideal Fermi gas and considering the accretion process through dark matter self-interaction from the surrounding halo. Non-annihilating heavy dark matter of mass greater than $1 \mathrm{GeV}$ is predicted to accumulate at the center of a neutron star leading it to a possible collapse [17]. The effect of this accumulation is observable only in cases where the annihilation cross section is extremely small $[18,19]$. The capture is fully efficient even for WIMP-to-nucleon cross sections (elastic or inelastic) as low as $10^{-18} \mathrm{mb}$. Moreover, a dark star of comparable mass may as well accrete neutron star matter to form a dark matter dominated neutron star. In addition to axions and WIMPs, a general class of dark matter candidates, called Macros, have been suggested that would have macroscopic size and mass [20].

Since dark matter interacts with normal baryonic matter through gravity, it is quite possible for white dwarfs and neutron stars to accrete dark matter and evolve to a dark matter admixed compact star $[12,15,17,21-26]$. The large baryonic density in compact stars increases the probability of dark matter capture within the star and eventually results in gravitational trapping. It may also be possible for dark matter alone to form gravitationally bound compact objects and thus mimic stellar mass black holes [27].

The hydrostatic equilibrium configuration of an admixture of degenerate dark matter and normal nuclear matter was studied by using a general relativistic two-fluid formalism taking non self-annihilating dark matter particles of a mass of $1 \mathrm{GeV}$. A new class of compact stars was predicted that consisted of a small normal matter core with radius of a few kilometers embedded in a $10 \mathrm{~km}$-sized dark matter halo [15].

Compact objects formed by non self-annihilating dark matter admixed with ordinary matter has been predicted with Earth-like masses and radii from few $\mathrm{km}$ to few hundred $\mathrm{km}$ for weakly interacting dark matter. For the strongly interacting dark matter case, dark compact planets are suggested to form with Jupiter-like masses and radii of few hundred $\mathrm{km}$ [28]. Possible implications of asymmetric fermionic dark matter for neutron stars have been studied that apply to various dark fermion models such as mirror matter models and to other models where the dark fermions have self-interactions [29].
Although dark matter particles can have only very weak interactions with standard model states, it is an intriguing possibility that they experience much stronger self-interactions and thereby alter the behavior of dark matter on astrophysical and cosmological scales in striking ways. Recent studies [30-35] have provided constraints on the dark matter self-interaction cross section. The constraints are based on the cusp-core problem and the "Too big to fail" problem of galaxies. According to them the dark matter self-interaction cross section per unit mass is about $0.1-100 \mathrm{~cm}^{2} / \mathrm{g} \sim 0.1-1$ barn $/ \mathrm{GeV}$, typical of the scale of strong interactions.

In this work we consider fermionic asymmetric dark matter (ADM) particles of a mass of $1 \mathrm{GeV}$ and the selfinteraction mediator mass of $100 \mathrm{MeV}$ (low mass implying strong interaction), mixed with rotating and non-rotating neutron stars. ADM, like ordinary baryonic matter, is charge asymmetric with only the dark baryon (or generally only the particle) excess remaining after the annihilation of most antiparticles after the Big Bang. Hence these ADM particles are non self-annihilating and behave like ordinary free particles. The gravitational stability and mass-radius relations of static, rigid and differentially rotating neutron stars mixed with fermionic ADM are calculated using the LORENE code [36]. It is important to note that we do not allow any phase transition of the nuclear matter and that the interaction between nuclear matter and dark matter is only through gravity.

\section{Equation of state of $\beta$-equilibrated nuclear matter}

The nuclear matter EoS is calculated using the isoscalar and the isovector [37,38] components of M3Y interaction along with density dependence. The density dependence of this DDM3Y effective interaction is completely determined from nuclear matter calculations. The equilibrium density of the nuclear matter is determined by minimizing the energy per nucleon. The energy variation of the zero range potential is treated accurately by allowing it to vary freely with the kinetic energy part $\epsilon^{k i n}$ of the energy per nucleon $\epsilon$ over the entire range of $\epsilon$. This is not only more plausible but also yields excellent results for the incompressibility $K_{\infty}$ of the SNM which does not suffer from the superluminosity problem [39].

In a Fermi gas model of interacting neutrons and protons with isospin asymmetry $X=\frac{\rho_{n}-\rho_{p}}{\rho_{n}+\rho_{p}}, \rho=\rho_{n}+\rho_{p}$, where $\rho_{n}, \rho_{p}$ and $\rho$ are the neutron, proton and nucleonic densities, respectively, the energy per nucleon for isospin asymmetric nuclear matter can be derived as [39]

$\epsilon(\rho, X)=\left[\frac{3 \hbar^{2} k_{F}^{2}}{10 m}\right] F(X)+\left(\frac{\rho J_{v} C}{2}\right)\left(1-\beta \rho^{n}\right)$ 
where $m$ is the nucleonic mass, $k_{F}=\left(1.5 \pi^{2} \rho\right)^{\frac{1}{3}}$ which equals the Fermi momentum in the case of SNM, the kinetic energy per nucleon $\epsilon^{k i n}=\left[\frac{3 \hbar^{2} k_{F}^{2}}{10 m}\right] F(X)$ with $F(X)=$ $\left[\frac{(1+X)^{5 / 3}+(1-X)^{5 / 3}}{2}\right]$ and $J_{v}=J_{v 00}+X^{2} J_{v 01}, J_{v 00}$ and $J_{v 01}$ represent the volume integrals of the isoscalar and the isovector parts of the M3Y interaction. The isoscalar $t_{00}^{M 3 Y}$ and the isovector $t_{01}^{M 3 Y}$ components of M3Y interaction potential are given by

$$
\begin{aligned}
t_{00}^{M 3 Y}(s, \epsilon)= & +7999 \frac{\exp (-4 s)}{4 s}-2134 \frac{\exp (-2.5 s)}{2.5 s} \\
& +J_{00}(1-\alpha \epsilon) \delta(s) \\
t_{01}^{M 3 Y}(s, \epsilon)= & -4886 \frac{\exp (-4 s)}{4 s}+1176 \frac{\exp (-2.5 s)}{2.5 s} \\
& +J_{01}(1-\alpha \epsilon) \delta(s)
\end{aligned}
$$

where $s$ represents the relative distance between two interacting nucleons, $J_{00}=-276 \mathrm{MeV} . \mathrm{fm}^{3}, J_{01}=+228 \mathrm{MeV} \mathrm{fm}^{3}$ and the energy dependence parameter $\alpha=0.005 \mathrm{MeV}^{-1}$. The Yukawa strengths were extracted by fitting the matrix elements in an oscillator basis to those elements of a G-matrix obtained with the Reid-Elliott soft core NN interaction. The ranges were selected to ensure OPEP tails in the relevant channels as well as a short-range part which simulates the $\sigma$ exchange process [40]. The density dependence is employed to account for the Pauli blocking effects and the higher order exchange effects [41]. Thus the DDM3Y effective NN interaction is given by $v_{0 i}(s, \rho, \epsilon)=t_{0 i}^{M 3 Y}(s, \epsilon) g(\rho)$ where the density dependence $g(\rho)=C\left(1-\beta \rho^{n}\right)$ [39] with $C$ and $\beta$ being the constants of the density dependence.

Equation (1) can be differentiated with respect to $\rho$ to yield an equation for $X=0$ :

$$
\begin{aligned}
\frac{\partial \epsilon}{\partial \rho}= & {\left[\frac{\hbar^{2} k_{F}^{2}}{5 m \rho}\right]+\frac{J_{v 00} C}{2}\left[1-(n+1) \beta \rho^{n}\right] } \\
& -\alpha J_{00} C\left[1-\beta \rho^{n}\right]\left[\frac{\hbar^{2} k_{F}^{2}}{10 m}\right] .
\end{aligned}
$$

The equilibrium density of the cold SNM is determined from the saturation condition. Then Eqs. (1) and (3) with the saturation condition $\frac{\partial \epsilon}{\partial \rho}=0$ at $\rho=\rho_{0}, \epsilon=\epsilon_{0}$ can be solved simultaneously for fixed values of the saturation energy per nucleon $\epsilon_{0}$ and the saturation density $\rho_{0}$ of the cold SNM to obtain the values of $\beta$ and $C$. The constants of the density dependence $\beta$ and $C$, thus obtained, are given by

$$
\beta=\frac{\left[(1-p)+\left(q-\frac{3 q}{p}\right)\right] \rho_{0}^{-n}}{\left[(3 n+1)-(n+1) p+\left(q-\frac{3 q}{p}\right)\right]}
$$

where $p=\frac{\left[10 m \epsilon_{0}\right]}{\left[\hbar^{2} k_{F_{0}}^{2}\right]}, q=\frac{2 \alpha \epsilon_{0} J_{00}}{J_{v 00}^{0}}, J_{v 00}^{0}=J_{v 00}\left(\epsilon_{0}^{k i n}\right)$ implying $J_{v 00}$ at $\epsilon^{k i n}=\epsilon_{0}^{k i n}$, the kinetic energy part of the saturation energy per nucleon of SNM, $k_{F_{0}}=\left[1.5 \pi^{2} \rho_{0}\right]^{1 / 3}$ and
$C=-\frac{\left[2 \hbar^{2} k_{F_{0}}^{2}\right]}{5 m J_{v 00}^{0} \rho_{0}\left[1-(n+1) \beta \rho_{0}^{n}-\frac{q \hbar^{2} k_{F_{0}}^{2}\left(1-\beta \rho_{0}^{n}\right)}{10 m \epsilon_{0}}\right]}$

respectively. It is quite obvious that the constants of the density dependence $C$ and $\beta$ obtained by this method depend on the saturation energy per nucleon $\epsilon_{0}$, the saturation density $\rho_{0}$, the index $n$ of the density dependent part and on the strengths of the M3Y interactions through the volume integral $J_{v 00}^{0}$.

The calculations are performed using the values of the saturation density $\rho_{0}=0.1533 \mathrm{fm}^{-3}$ [42] and the saturation energy per nucleon $\epsilon_{0}=-15.26 \mathrm{MeV}$ [43] for the SNM obtained from the coefficient of the volume term of the Bethe-Weizsäcker mass formula which is evaluated by fitting the recent experimental and estimated atomic mass excesses from the Audi-Wapstra-Thibault atomic mass table [44] by minimizing the mean square deviation incorporating correction for the electronic binding energy [45]. In a similar recent work, addressing the surface symmetry energy term, the Wigner term, the shell correction and the proton form factor correction to the Coulomb energy, the $a_{v}$ turns out to be $15.4496 \mathrm{MeV}$ and when the $A^{0}$ and $A^{1 / 3}$ terms are also included it becomes $14.8497 \mathrm{MeV}$ [46]. Using the usual values of $\alpha=0.005 \mathrm{MeV}^{-1}$ for the parameter of the energy dependence of the zero range potential and $n=2 / 3$, the values obtained for the constants of density dependence $C$ and $\beta$ and the SNM incompressibility $K_{\infty}$ are $2.2497,1.5934$ $\mathrm{fm}^{2}$ and $274.7 \mathrm{MeV}$, respectively. The saturation energy per nucleon is the volume energy coefficient and the value of $-15.26 \pm 0.52 \mathrm{MeV}$ covers, more or less, the entire range of values obtained for $a_{v}$ for which now we have the values of $C=2.2497 \pm 0.0420, \beta=1.5934 \pm 0.0085 \mathrm{fm}^{2}$ and the SNM incompressibility $K_{\infty}=274.7 \pm 7.4 \mathrm{MeV}$.

The symmetric nuclear matter incompressibility $K_{\infty}$, the nuclear symmetry energy at saturation density $E_{\mathrm{sym}}\left(\rho_{0}\right)$, the slope $L$ and the isospin dependent part $K_{\tau}$ of the isobaric incompressibility are also tabulated in Table 1 , since these are all in excellent agreement with the recently extracted constraints from the measured isotopic dependence of the giant monopole resonances in even-A Sn isotopes [47], from the neutron skin thickness of nuclei and from analyses of experimental data on isospin diffusion and isotopic scaling in intermediate energy heavy-ion collisions.

The calculations for masses and radii are performed using the EoS covering the crustal region of a compact star which are the Feynman-Metropolis-Teller (FMT) [48], BaymPethick-Sutherland (BPS) [49] and Baym-Bethe-Pethick (BBP) [50] cases up to number density of $0.0582 \mathrm{fm}^{-3}$ and $\beta$-equilibrated neutron star matter beyond this value. Figures 1 and 2 represent the mass-central density and mass-radius plots, respectively, for slowly rotating pure neutron stars with 
Table 1 Results of present calculations for $n=\frac{2}{3}$ of symmetric nuclear matter incompressibility $K_{\infty}$, nuclear symmetry energy at saturation density $E_{\text {sym }}\left(\rho_{0}\right)$, the slope $L$ and the isospin dependent part $K_{\tau}$ of the isobaric incompressibility (all in $\mathrm{MeV}$ ) $[54,55]$ are tabulated

\begin{tabular}{llll}
\hline$K_{\infty}$ & $E_{\text {sym }}\left(\rho_{0}\right)$ & $L$ & $K_{\tau}$ \\
\hline $274.7 \pm 7.4$ & $30.71 \pm 0.26$ & $45.11 \pm 0.02$ & $-408.97 \pm 3.01$
\end{tabular}

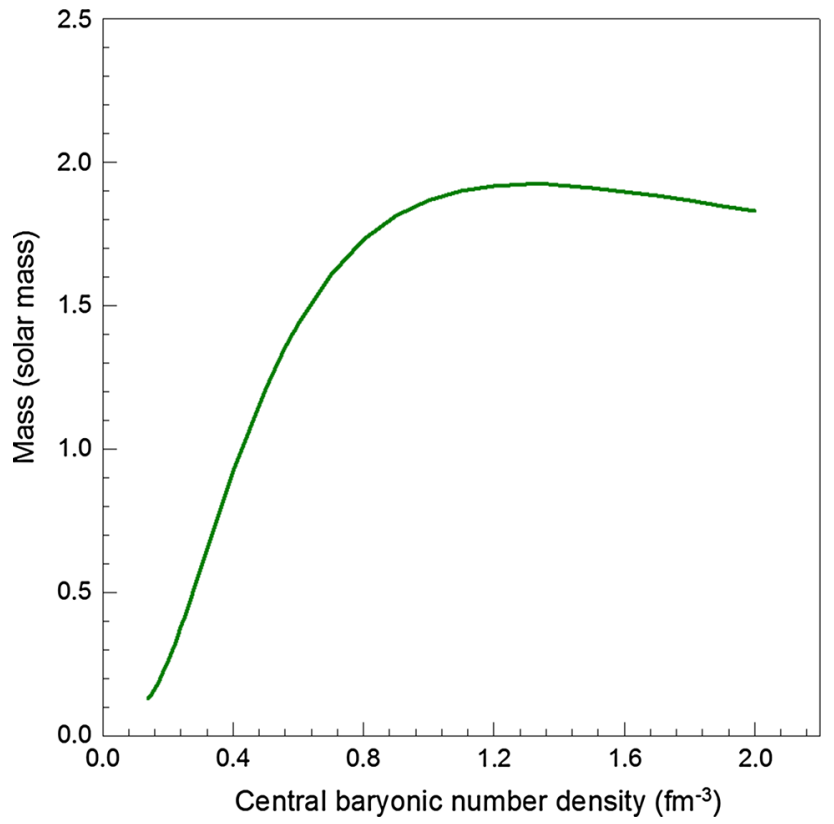

Fig. 1 Mass vs. central baryonic density plot of slowly rotating neutron stars for the DDM3Y EoS

DDM3Y EoS. The maximum mass goes to $1.9227 \mathrm{M}_{\odot}$ with a radius of $9.7559 \mathrm{~km}$ [51-53].

\section{Equation of state of non-interacting fermionic asymmetric dark matter}

We consider the non-interacting fermionic ADM to be a completely degenerate free Fermi gas of particle mass $m_{\chi}$ at zero temperature. By the Pauli exclusion principle no quantum state can be occupied by more than one fermion with an identical set of quantum numbers. Thus a non-interacting Fermi gas, unlike a Bose gas, is prohibited from condensing into a Bose-Einstein condensate. The total energy of the Fermi gas at absolute zero is larger than the sum of the single-particle ground states because the Pauli principle implies a degeneracy pressure that keeps fermions separated and moving.

The non-interacting assembly of fermions at zero temperature exerts pressure because of kinetic energy from different states filled up to Fermi level. Since the pressure is the force per unit area, which means the rate of momentum transfer per unit area, it is given by

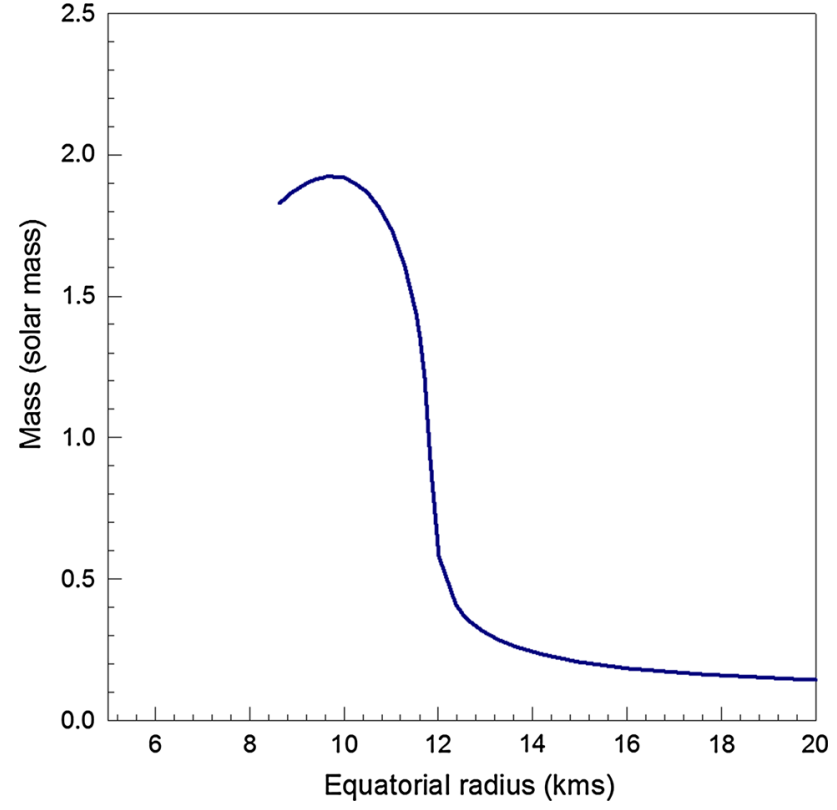

Fig. 2 Mass-equatorial radius plot of slowly rotating neutron stars for the DDM3Y EoS

$P_{\chi}=\frac{1}{3} \int p v n_{p} d^{3} p=\frac{1}{3} \int \frac{p^{2} c^{2}}{\sqrt{\left(p^{2} c^{2}+m_{\chi}^{2} c^{4}\right)}} n_{p} d^{3} p$

where $m_{\chi}$ is the rest mass of dark particles, $v$ is the velocity of the particles with momentum $\vec{p}$ and $n_{p} d^{3} p$ is the number of particles per unit volume having momenta between $\vec{p}$ and $\vec{p}+\mathrm{d} \vec{p}$. The factor $\frac{1}{3}$ accounts for the fact that, on average, only $\frac{1}{3}$ of total particles $n_{p} d^{3} p$ are moving in a particular direction. For fermions having spin $\frac{1}{2}$, degeneracy $=2$, $n_{p} d^{3} p=\frac{8 \pi p^{2} d p}{h^{3}}$ and hence the number density $n_{\chi}$ is given by

$n_{\chi}=\int_{0}^{p_{F}} n_{p} d^{3} p=\frac{8 \pi p_{F}^{3}}{3 h^{3}}=\frac{x_{F}^{3}}{3 \pi^{2} \lambda_{\chi}^{3}}$

where $p_{F}$ is the Fermi momentum, which is the maximum momentum possible at zero temperature, $x_{F}=\frac{p_{F}}{m_{\chi} c}$ is a dimensionless quantity and $\lambda_{\chi}=\frac{\hbar}{m_{\chi} c}$ is the Compton wavelength. The energy density $\varepsilon_{\chi}$ is given by

$\varepsilon_{\chi}=\int_{0}^{p_{F}} E n_{p} d^{3} p=\int_{0}^{p_{F}} \sqrt{\left(p^{2} c^{2}+m_{\chi}^{2} c^{4}\right)} \frac{8 \pi p^{2} d p}{h^{3}}$,

which, along with Eq. (7), turns out upon integration to be

$\varepsilon_{\chi}=\frac{m_{\chi} c^{2}}{\lambda_{\chi}^{3}} \chi\left(x_{F}\right) ; \quad P_{\chi}=\frac{m_{\chi} c^{2}}{\lambda_{\chi}^{3}} \phi\left(x_{F}\right)$, 
where

$\chi(x)=\frac{1}{8 \pi^{2}}\left[x \sqrt{1+x^{2}}\left(1+2 x^{2}\right)-\ln \left(x+\sqrt{1+x^{2}}\right)\right]$

and

$\phi(x)=\frac{1}{8 \pi^{2}}\left[x \sqrt{1+x^{2}}\left(\frac{2 x^{2}}{3}-1\right)+\ln \left(x+\sqrt{1+x^{2}}\right)\right]$.

\section{Equation of state of strongly self-interacting fermionic asymmetric dark matter}

In order to calculate the EoS of a strongly interacting fermionic ADM we turn to massive vector field theory similar to the meson exchange of the nuclear interaction. The Lagrangian density (in natural units) of a massive vector field is given by

$\mathcal{L}=-\frac{1}{4} F_{\mu \nu} F^{\mu \nu}+\frac{1}{2} m_{I}^{2} A_{\mu} A^{\mu}-j_{\mu} A^{\mu}$

where $F_{\mu \nu}=\partial_{\mu} A_{\nu}-\partial_{\nu} A_{\mu}, A^{\mu}$ is the four-vector field, $j^{\mu}$ is the four-current and $m_{I}$ is the mass of the field quanta. The equation of motion is given by

$\left(\partial_{\nu} \partial^{\nu}+m_{I}^{2}\right) A^{\mu}=j^{\mu}$.

Now considering a charge of magnitude $g$ at rest at the origin we have

$j^{0}=g \delta^{3}(\vec{x}) \quad \vec{j}=0$.

Substituting the above in the right side of Eq. (14) and also noting that $A^{0}=V$ and $\vec{A}=0$ we finally get

$\left(\nabla^{2}-m_{I}^{2}\right) V=-g \delta^{3}(\vec{x})$

whose solution is the Yukawa potential:

$V(r)=g \frac{\mathrm{e}^{-m_{I} r}}{4 \pi r}$.

Hence the potential energy of two like charges of magnitude $g$ is

$V_{12}(r)=g^{2} \frac{\mathrm{e}^{-m_{I} r}}{4 \pi r}$

and is repulsive in nature.
To proceed to the EoS, we calculate the total energy of a system of particles classically by summing over the interactions of all pairs of particles. To facilitate the calculation, we assume that the macroscopic assembly is uniformly distributed, thereby neglecting the influence of the interaction on the mean interparticle separation. In other words, we ignore any correlations between particle positions due to their mutual interaction. Finally, we assume that the number of particles is sufficiently large so that we can replace sums by integrals and the characteristic size of the assembly $R$ satisfies $R \gg 1 / m_{I}$ [56].

The total Yukawa potential energy of a system of $N$ particles in volume $\Omega$ is

$E_{\Omega}=\frac{1}{2} \sum_{i \neq j} V_{i j}=\frac{1}{2} n^{2} g^{2} \iint \frac{e^{-m_{I} r_{i j}}}{4 \pi r_{i j}} \mathrm{~d} \Omega_{i} \mathrm{~d} \Omega_{j}$,

where $n$ is the number density.

Choosing one particle at the origin and integrating to infinity (ignoring surface terms) we find

$E_{\Omega}=\frac{1}{2 m_{I}^{2}} n^{2} g^{2} \Omega$,

so that the interaction energy density can be written as

$\varepsilon_{\text {int }}=\frac{E_{\Omega}}{\Omega}=\frac{1}{2 m_{I}^{2}} n^{2} g^{2}$.

Now putting $g^{2} / 2=1$ for convenience, $x_{f}=k_{f} / m_{\chi}$, where $m_{\chi}$ is the rest mass of the dark matter particle and using the relation $k_{f}=\left(3 \pi^{2} n\right)^{1 / 3}$ we get putting back $\hbar$ and $c$

$\varepsilon_{\text {int }}=\left(\frac{1}{3 \pi^{2}}\right)^{2} \frac{x_{f}^{6} m_{\chi}^{6}}{(\hbar c)^{3} m_{I}^{2}}$,

where $m_{\chi}$ and $m_{I}$ are expressed in MeV.

The pressure due to the interacting energy density can be computed with the help of the thermodynamic relation $P_{\text {int }}=n^{2} \frac{\mathrm{d}}{\mathrm{d} n}\left(\frac{\varepsilon_{\text {int }}}{n}\right)$, which yields

$P_{\text {int }}=\left(\frac{1}{3 \pi^{2}}\right)^{2} \frac{x_{f}^{6} m_{\chi}^{6}}{(\hbar c)^{3} m_{I}^{2}}$.

Hence the total energy density and pressure of selfinteracting dark matter particles are given by

$$
\begin{aligned}
& \varepsilon_{\chi \mathrm{int}}=\varepsilon_{\chi}+\varepsilon_{\mathrm{int}}=\frac{m_{\chi}}{\lambda_{\chi}^{3}} \chi\left(x_{F}\right)+\left(\frac{1}{3 \pi^{2}}\right)^{2} \frac{x_{f}^{6} m_{\chi}^{6}}{(\hbar c)^{3} m_{I}^{2}}, \\
& P_{\chi \mathrm{int}}=P_{\chi}+P_{\mathrm{int}}=\frac{m_{\chi}}{\lambda_{\chi}^{3}} \phi\left(x_{F}\right)+\left(\frac{1}{3 \pi^{2}}\right)^{2} \frac{x_{f}^{6} m_{\chi}^{6}}{(\hbar c)^{3} m_{I}^{2}} .
\end{aligned}
$$




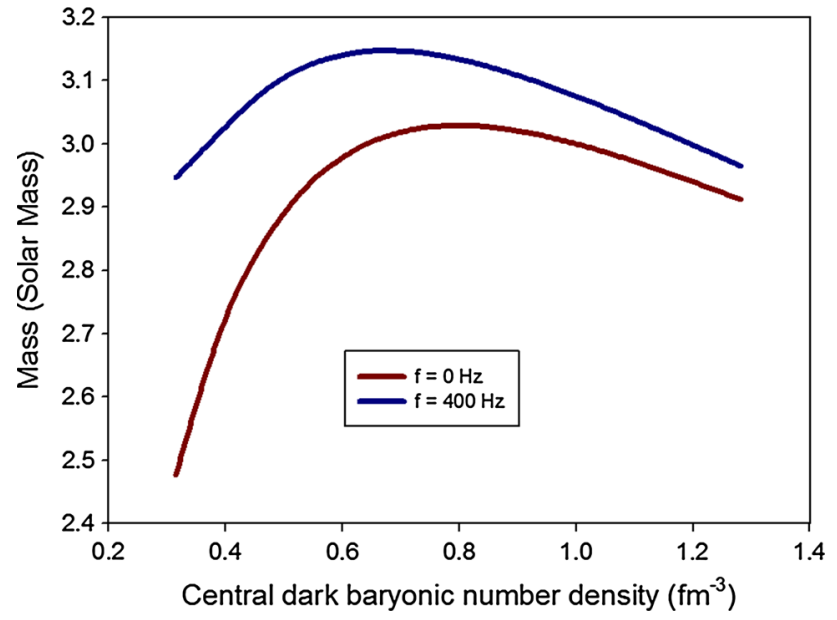

Fig. 3 Plots of mass vs. central density for static and rotating fermionic asymmetric dark matter stars

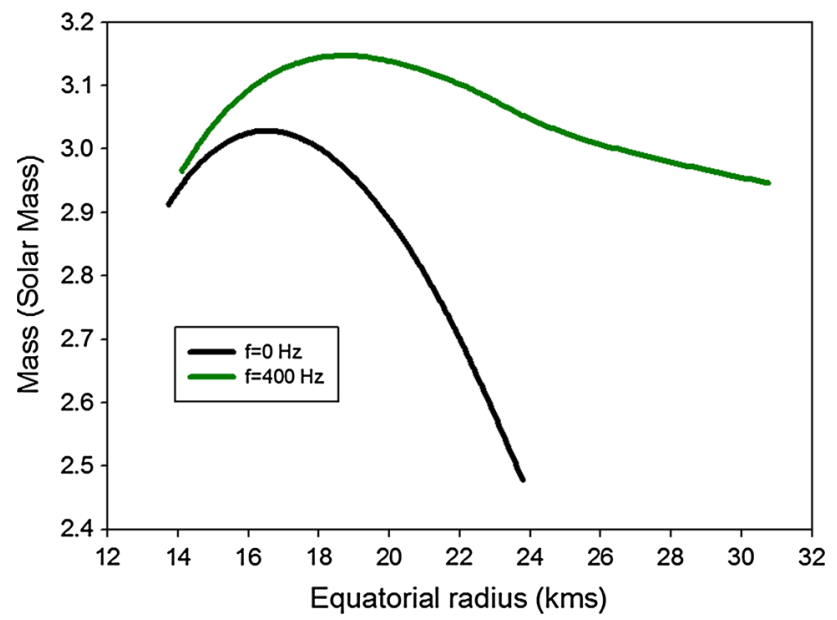

Fig. 4 Mass-equatorial radius plots for static and rotating fermionic asymmetric dark matter stars

The mass of the exchange boson determines the strength and the range of the interaction, implying that the lower the mass is, the stronger the interaction. For non-interacting dark matter $m_{I}$ is infinite and second terms in the above equations are absent.

Figures 3 and 4 depict the plots of mass vs. central dark matter density and mass vs. equatorial radius, respectively, for static and rotating stars, using a self-interacting dark matter EoS. We see that the maximum mass for non-rotating stars goes to $3.0279 \mathrm{M}_{\odot}$ with a radius of $16.2349 \mathrm{~km}$ and that the maximum mass for rotating stars goes to $3.1460 \mathrm{M}_{\odot}$ with equatorial radius of $19.2173 \mathrm{~km}$. Now, if we take the dark matter particle mass $m_{\chi}$ to be $0.5 \mathrm{GeV}$ the maximum mass goes to $\sim 12.6 \mathrm{M}_{\odot}$ using the relation mass $\propto 1 / m_{\chi}^{2}$ [24], thus mimicking stellar mass black holes.

\section{Two-fluid TOV equation}

We consider two ideal fluids-the nuclear matter and fermionic dark matter with the above two EoSs coupled gravitationally to form the structure of the mixed neutron star. The energy-momentum tensor of the mixed fluid can be written as $[29,57]$

$$
\begin{aligned}
T^{\mu \nu}= & T_{\text {nuc }}^{\mu \nu}+T_{\text {dark }}^{\mu \nu}=\left(\varepsilon_{\text {nuc }}+P_{\text {nuc }}\right) u_{1}^{\mu} u_{1}^{\nu}-P_{\text {nuc }} g^{\mu \nu} \\
& +\left(\varepsilon_{\text {dark }}+P_{\text {dark }}\right) u_{2}^{\mu} u_{2}^{\nu}-P_{\text {dark }} g^{\mu \nu}
\end{aligned}
$$

where $u_{1}^{\mu}, \varepsilon_{\text {nuc }}$ and $P_{\text {nuc }}$ are the 4-velocity, energy density and pressure of nuclear matter, respectively, while the corresponding quantities in the second term are for dark matter.

For a non-rotating case the metric is spherically symmetric and the hydrostatic equations of the two fluids can be written as coupled two-fluid Tolman-Oppenheimer-Volkoff (TOV) equations,

$$
\begin{aligned}
\frac{\mathrm{d} P_{\mathrm{nuc}}(r)}{\mathrm{d} r}= & -\frac{G M(r) \rho_{\mathrm{nuc}}(r)}{r^{2}}\left(1+\frac{P_{\mathrm{nuc}}}{\varepsilon_{\mathrm{nuc}}}\right) \\
& \times\left(1+\frac{4 \pi r^{3}\left(P_{\mathrm{nuc}}+P_{\mathrm{dark}}\right)}{M(r) c^{2}}\right)\left(1-\frac{2 G M(r)}{r c^{2}}\right)^{-1} \\
\frac{\mathrm{d} P_{\mathrm{dark}}(r)}{\mathrm{d} r}= & -\frac{G M(r) \rho_{\mathrm{dark}}(r)}{r^{2}}\left(1+\frac{P_{\mathrm{dark}}}{\varepsilon_{\mathrm{dark}}}\right) \\
& \times\left(1+\frac{4 \pi r^{3}\left(P_{\mathrm{nuc}}+P_{\mathrm{dark}}\right)}{M(r) c^{2}}\right)\left(1-\frac{2 G M(r)}{r c^{2}}\right)^{-1} \\
\frac{\mathrm{d} M_{\mathrm{nuc}}(r)}{\mathrm{d} r}= & 4 \pi r^{2} \rho_{\text {nuc }}(r), \\
\frac{\mathrm{d} M_{\mathrm{dark}}(r)}{\mathrm{d} r}= & 4 \pi r^{2} \rho_{\text {dark }}(r), \\
M(r)= & M_{\mathrm{nuc}}(r)+M_{\mathrm{dark}}(r),
\end{aligned}
$$

where $\rho_{\text {nuc }}=\varepsilon_{\text {nuc }} / c^{2}, M_{\text {nuc }}$ is the mass density and the total mass of nuclear matter, while the corresponding quantities in the second equation are for dark matter. $M(r)$ is the total mass of nuclear and dark matter.

\section{Theoretical calculations}

The mass-radius relationship of non-rotating, rigidly rotating and differentially rotating neutron stars admixed with dark matter is calculated using the LORENE code. The nuclear matter and dark matter EoSs are fitted to a polytropic form $P=K \rho^{\gamma}$ where $P$ is the pressure, $\rho$ is the mass density, $K$ the polytropic constant and $\gamma$ the polytropic index for the corresponding fluid. For interacting nuclear matter $\gamma=2.03$ and $K=5.65283 \times 10^{35}$ in C.G.S. units. For interacting dark matter $\gamma=1.97562$ and $K=1.33404 \times 10^{36}$ in C.G.S. units. We take the dark matter particle mass to be 1 $\mathrm{GeV}$ and the exchange boson mass $m_{I}=100 \mathrm{MeV}$, typical of the strong interaction. First, we assume the dark matter central enthalpy to be $0.24 c^{2}$ (fixed) and vary the nuclear 
matter central enthalpy for static, rigidly rotating and differentially rotating configurations and next we reverse the roles of nuclear and dark matter.

\section{Results and discussions}

In Fig. 5 the plots of total mass vs. equatorial radius of static, rigidly and differentially rotating neutron stars mixed with fermionic self-interacting dark matter are shown for fixed dark matter central enthalpy $\left(0.24 c^{2}\right)$ and varying nuclear matter central enthalpies. In Fig. 6 the corresponding plots of mass vs. central baryonic number density are shown. The maximum mass of the neutron star mixed with strongly self-

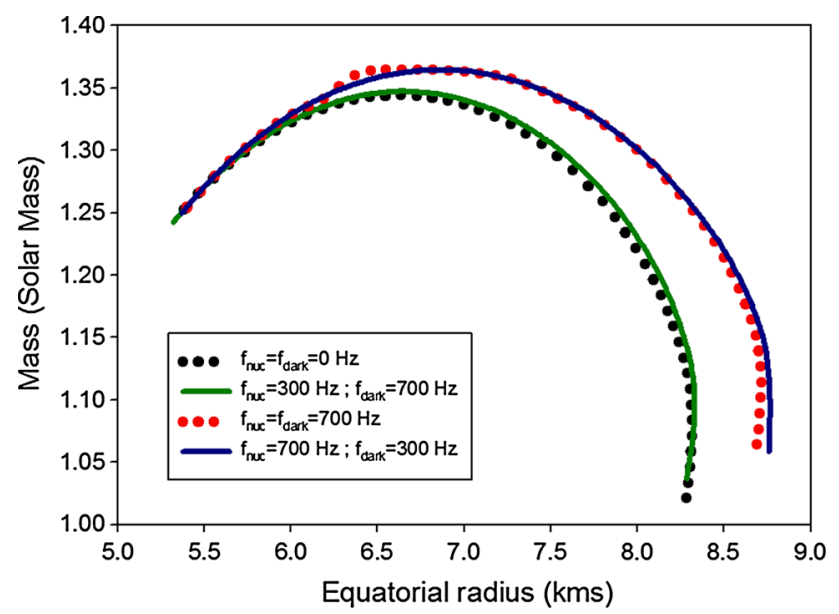

Fig. 5 Plots of total mass vs. equatorial radius of static, rigidly rotating and differentially rotating neutron stars mixed with interacting fermionic asymmetric dark matter with fixed dark matter central enthalpy $\left(0.24 c^{2}\right)$ and varying nuclear matter central enthalpies

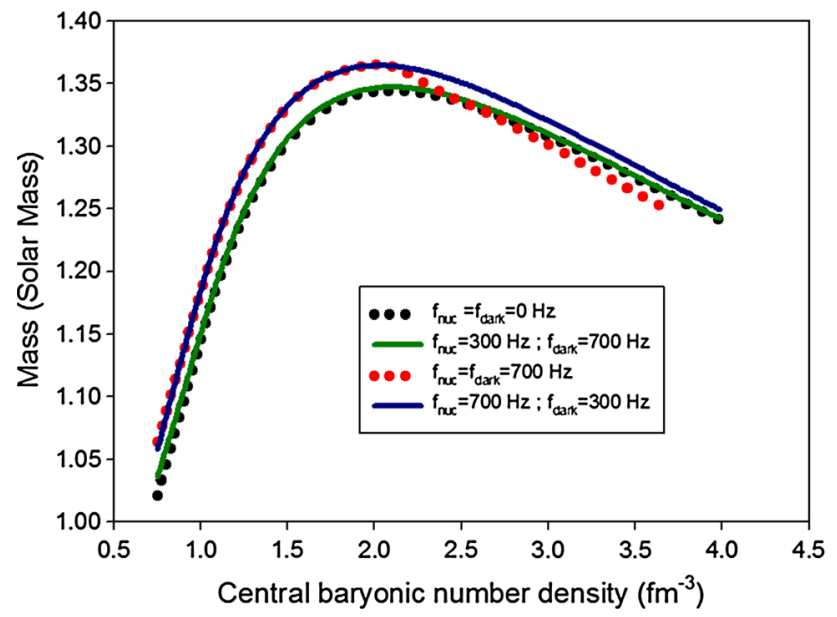

Fig. 6 Plots of total mass vs. central baryonic density of static, rigidly rotating and differentially rotating neutron stars mixed with selfinteracting fermionic asymmetric dark matter with fixed dark matter central enthalpy $\left(0.24 c^{2}\right)$ and varying nuclear matter central enthalpies interacting dark matter goes to $1.3640 \mathrm{M}_{\odot}$ with a corresponding radius of $6.7523 \mathrm{~km}$ for differential rotation (frequency of dark matter to be $300 \mathrm{~Hz}$ and that of nuclear matter to be $700 \mathrm{~Hz}$ ) as shown in Fig. 5. From Fig. 6 we see that the corresponding central baryonic number density is $2.1060 \mathrm{fm}^{-3}$. In this case the maximum gravitational mass is $1.3640 \mathrm{M}_{\odot}$, the corresponding matter mass is $1.5024 \mathrm{M}_{\odot}$, which is constituted of nuclear matter $1.4719 \mathrm{M}_{\odot}$ and dark matter $0.0305 \mathrm{M}_{\odot}$.

In Fig. 7 the plots of total mass vs. equatorial radius of static, rigidly and differentially rotating neutron stars mixed with fermionic self-interacting dark matter are shown for fixed nuclear matter central enthalpy $\left(0.24 c^{2}\right)$ and varying dark matter central enthalpies. In Fig. 8 the corresponding

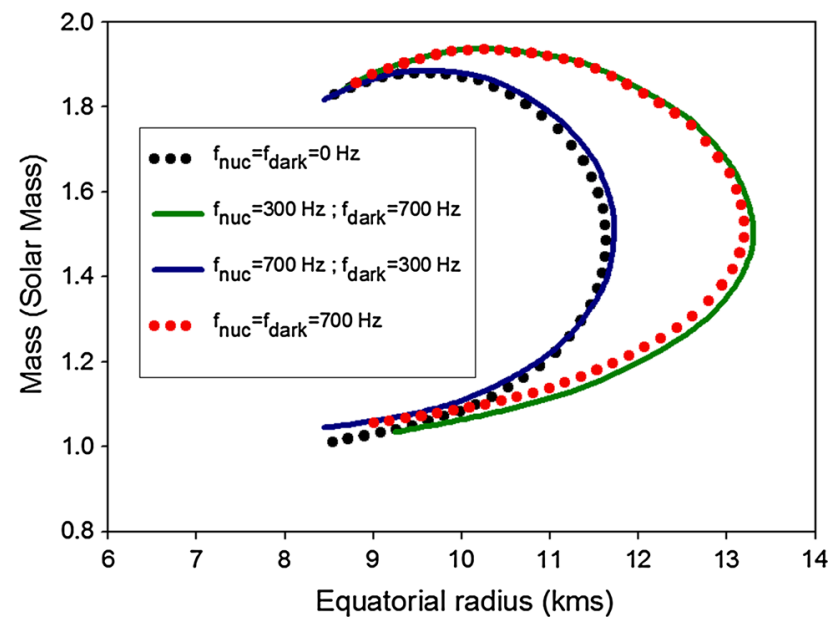

Fig. 7 Plots of total mass vs. equatorial radius of static, rigidly rotating and differentially rotating neutron stars mixed with interacting fermionic asymmetric dark matter with fixed nuclear matter central enthalpy $\left(0.24 c^{2}\right)$ and varying dark matter central enthalpies

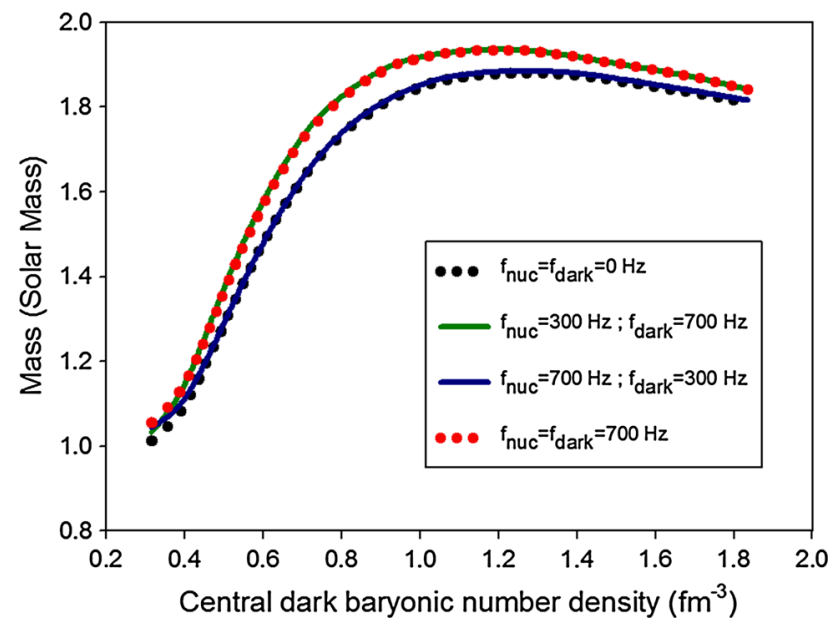

Fig. 8 Plots of total mass vs. central dark matter density of static, rigidly rotating and differentially rotating neutron stars mixed with selfinteracting fermionic asymmetric dark matter with fixed nuclear matter central enthalpy $\left(0.24 c^{2}\right)$ and varying dark matter central enthalpies 
plots of mass vs. central dark baryonic number density are shown. In this case the maximum mass goes to $1.9355 \mathrm{M}_{\odot}$ with a corresponding radius of $10.3717 \mathrm{~km}$ for differential rotation (frequency of dark matter to be $700 \mathrm{~Hz}$ and that of nuclear matter to be $300 \mathrm{~Hz}$ ) as shown in Fig. 7. From Fig. 8 we see that the corresponding central dark baryonic number density is $1.1605 \mathrm{fm}^{-3}$. In this case the maximum gravitational mass is $1.9355 \mathrm{M}_{\odot}$, the corresponding matter mass is $2.1105 \mathrm{M}_{\odot}$, which constitutes of nuclear matter $0.1179 \mathrm{M}_{\odot}$ and dark matter $1.9926 \mathrm{M}_{\odot}$.

It is seen that the polytropic indices $\gamma$ for nuclear and selfinteracting dark matter EoSs are approximately equal. However, the polytropic coefficient $K$ for dark matter is about 2.5 times larger than that of nuclear matter making dark matter EoS stiffer. Consequently, configurations of stars with varying dark matter central enthalpy with fixed nuclear matter central enthalpy are more massive than those obtained in the reverse case.

From Fig. 7 we see that the dark matter dominated neutron star behaves differently than the nuclear matter dominated one as shown in Fig. 5. In Fig. 7, the plots of low mass neutron stars admixed with dark matter typically show the characteristics similar to low mass self-bound strange stars. This is because of the very strong two-body repulsive interactions of dark matter, which is dominant in the configuration of Fig. 7, which counteract the gravity effectively for the low mass region and make the radius much smaller compared to the pure neutron star of a similar mass (vide Fig. 2). Thus, while the nuclear matter dominance induces gravitational binding, a dark matter dominant low mass neutron star becomes gravitationally bound at a much smaller radius.

The maximum mass for non-rotating dark matter stars goes to $3.0279 \mathrm{M}_{\odot}$ with a radius of $16.2349 \mathrm{~km}$ for particle mass $m_{\chi}=1 \mathrm{GeV}$. For rotating stars the maximum mass goes to $3.1460 \mathrm{M}_{\odot}$ with a radius of $19.2173 \mathrm{~km}$. However, if one takes $m_{\chi}$ to be $0.5 \mathrm{GeV}$, then the maximum mass goes to $\sim 12.6 \mathrm{M}_{\odot}$ using the relation mass $\propto 1 / m_{\chi}^{2}$ [24], thus mimicking stellar mass black holes.

\section{Summary and conclusions}

In this work we consider fermionic asymmetric dark matter (ADM) particles of mass $1 \mathrm{GeV}$ and the self-interaction mediator mass of $100 \mathrm{MeV}$ (low mass implying strong interaction), mixed with rotating and non-rotating neutron stars. These ADM particles are non self-annihilating and behave like ordinary free particles. We have shown that a massive exotic neutron star with a strong two-body self-interacting fermionic dark matter is gravitationally stable with equal or unequal rotational frequencies of the two fluids. This provides an alternative scenario for the existence of $\sim 2 \mathrm{M}_{\odot}$ neutron stars with 'stiff' equations of state.
The mass-radius relations of pure hadronic stars mixed with self-interacting fermionic asymmetric dark matter have been obtained using the LORENE code. In the case of pure dark matter stars consisting of less massive dark particles we see that the maximum masses are comparable to those of stellar mass black holes. In the case of hadronic stars mixed with dark matter we considered three different configurationsstatic, rigid rotation and differential rotation of nuclear matter and dark matter fluids. From the results we conclude that for the dark matter dominated configurations the masses are higher, viz. for the static case the maximum masses of these hybrid stars can reach up to $\sim 1.88 \mathrm{M}_{\odot}$ with corresponding radii $\sim 9.5 \mathrm{~km}$, whereas in the rigid and differential rotational cases the maximum masses of these hybrid stars can reach up to $\sim 1.94 \mathrm{M}_{\odot}$ with corresponding equatorial radii $\sim 10.4$ $\mathrm{km}$.

We also find that the dark matter dominated neutron star behaves differently from the nuclear matter dominated one, which shows characteristics similar to low mass self-bound strange stars. This is because of the very strong two-body repulsive interactions of dark matter which is dominant in the low mass region where it counteracts gravity effectively to make radius much smaller. Thus, while the nuclear matter dominance induces gravitational binding, dark matter dominant low mass neutron star becomes more compact. However, if the dark matter particle mass is small compared to the nucleon mass, the maximum mass may well be above $2 \mathrm{M}_{\odot}$, provided no phase transition from nuclear to quark matter occurs.

In the past, phase transition and the possible existence of a pion condensate or quark matter inside compact stars have been studied extensively [58-62]. The phase transition from baryonic matter to quark matter is determined by the transition density. It is observed that a deconfinement transition inside a neutron star causes reduction in its mass. It would be interesting to find the effect of dark matter on the compact hybrid stars (baryonic matter with quark core), mixed with self-interacting fermionic asymmetric dark matter. Such an effect cannot be predicted a priori without full calculations and we leave it for future investigation.

Open Access This article is distributed under the terms of the Creative Commons Attribution 4.0 International License (http://creativecomm ons.org/licenses/by/4.0/), which permits unrestricted use, distribution, and reproduction in any medium, provided you give appropriate credit to the original author(s) and the source, provide a link to the Creative Commons license, and indicate if changes were made.

Funded by $\mathrm{SCOAP}^{3}$.

\section{References}

1. Y. Utsumi et al., Astrophys. J. 833, 156 (2016)

2. G. Bertone, D. Hooper, J. Silk, Phys. Rep. 405, 279 (2005) 
3. G.R. Blumenthal, S.M. Faber, J.R. Primack, M.J. Rees, Nature 311, 517 (1984)

4. S. King, A. Merle, JCAP 016, 1208 (2012)

5. K.M. Zurek, Phys. Rep. 537, 91 (2014)

6. C.S. Frenk, S.D.M. White, Ann. Phys. 524, 507 (2012)

7. D. Spolyar, K. Freese, P. Gondolo, Phys. Rev. Lett. 100, 051101 (2008)

8. A.H. Guth, M.P. Hertzberg, C. Prescod-Weinstein, Phys. Rev. D 92, 103513 (2015)

9. X. Li, T. Harko, K. Cheng, JCAP 06, 001 (2012)

10. G. Steigman, C. Sarazin, H. Quintana, J. Faulkner, Astron. J. 06, 1050 (1978)

11. I. Goldman, S. Nussinov, Phys. Rev. D 40, 3221 (1989)

12. G. Bertone, M. Fairbairn, Phys. Rev. D 77, 043515 (2008)

13. M.T. Frandsen, S. Sarkar, Phys. Rev. Lett. 105, 011301 (2010)

14. P. Ciarcelluti, F. Sandin, Phys. Lett. B 695, 19 (2011)

15. S.-C. Leung, M.-C. Chu, L.-M. Lin, Phys. Rev. D 84, 107301 (2011)

16. X.Y. Li, F.Y. Wang, K.S. Cheng, JCAP 10, 031 (2012)

17. A. de Lavallaz, M. Fairbairn, Phys. Rev. D 81, 123521 (2010)

18. C. Kouvaris, P. Tinyakov, Phys. Rev. D 82, 063531 (2010)

19. C. Kouvaris, P. Tinyakov, Phys. Rev. D 83, 083512 (2011)

20. D.M. Jacobs, G.D. Starkman, B.W. Lynn, MNRAS 450, 3418 (2015)

21. C. Kouvaris, Phys. Rev. D 77, 023006 (2008)

22. M. McCullough, M. Fairbairn, Phys. Rev. D 81, 083520 (2010)

23. M.A. Pérez-García, J. Silk, Phys. Lett. B 711, 6 (2012)

24. G. Narain, J. Schaffner-Bielich, I.N. Mishustin, Phys. Rev. D 74, 063003 (2006)

25. A. Li, F. Huang, X. Ren-Xin, Astropart. Phys. 37, 70 (2012)

26. Q.-F. Xiang, W.-Z. Jiang, D.-R. Zhang, R.-Y. Yang, Phys. Rev. C 89, 025803 (2014)

27. M.R.S. Hawkins, Mon. Not. R. Astron. Soc. 415, 2744 (2011)

28. L. Tolos, J. Schaffner-Bielich, Phys. Rev. D 92, 123002 (2015)

29. I. Goldman, R.N. Mohapatra, S. Nussinov, D. Rosenbaum, V. Teplitz, Phys. Lett. B 725, 200 (2013)

30. J. Pollack, D.N. Spergel, P.J. Steinhardt, Astrophys. J. 804, 131 (2015)

31. Y. Hochberg, E. Kuflik, H. Murayama, T. Volansky, J.G. Wacker, Phys. Rev. Lett. 115, 021301 (2015)

32. C.-S. Chen, G.-L. Lin, Y.-H. Lin, JCAP 01, 013 (2016)

33. J.M. Cline, Z. Liu, G.D. Moore, W. Xue, Phys. Rev. D 90, 015023 (2014)

34. K.K. Boddy, J.L. Feng, M. Kaplinghat, M.P. Tim, Tait. Phys. Rev. D 89, 115017 (2014)
35. M. Rocha, A.H.G. Peter, J.S. Bullock, M. Kaplinghat, S. GarrisonKimmel, J. Oñorbe, L.A. Moustakas, MNRAS 430, 81 (2013)

36. E. Gourgoulhon (2011). arXiv:1003.5015v2

37. A.M. Lane, Nucl. Phys. 35, 676 (1962)

38. G.R. Satchler, Int. series of monographs on Physics. Direct nuclear reactions (Oxford University Press, Oxford, 1983), p. 470

39. D.N. Basu, P.R. Chowdhury, C. Samanta, Nucl. Phys. A 811, 140 (2008)

40. G. Bertsch, J. Borysowicz, H. McManus, W.G. Love, Nucl. Phys. A 284, 399 (1977)

41. G.R. Satchler, W.G. Love, Phys. Rep. 55, 183 (1979)

42. C. Samanta, D. Bandyopadhyay, J.N. De, Phys. Lett. B 217, 381 (1989)

43. P.R. Chowdhury, D.N. Basu, Acta Phys. Pol. B 37, 1833 (2006)

44. G. Audi, A.H. Wapstra, C. Thibault, Nucl. Phys. A 729, 337 (2003)

45. D. Lunney, J.M. Pearson, C. Thibault, Rev. Mod. Phys. 75, 1021 (2003)

46. G. Royer, C. Gautier, Phys. Rev. C 73, 067302 (2006)

47. T. Li, U. Garg, Y. Liu et al., Phys. Rev. Lett. 99, 162503 (2007)

48. R.P. Feynman, N. Metropolis, E. Teller, Phys. Rev. 75, 1561 (1949)

49. G. Baym, C.J. Pethick, P. Sutherland, Astrophys. J. 170, 299 (1971)

50. G. Baym, H.A. Bethe, C.J. Pethick, Nucl. Phys. A 175, 225 (1971)

51. D. Atta, S. Mukhopadhyay, D.N. Basu, Indian J. Phys. 91, 235 (2017)

52. D. Atta, D.N. Basu, Phys. Rev. C 90, 035802 (2014)

53. W.M. Seif, D.N. Basu, Phys. Rev. C 89, 028801 (2014)

54. P.R. Chowdhury, D.N. Basu, C. Samanta, Phys. Rev. C 80, 011305(R) (2009)

55. D.N. Basu, P.R. Chowdhury, C. Samanta, Phys. Rev. C 80, 057304 (2009)

56. S.L. Shapiro, S.A. Teukolsky, Black Holes, White Dwarfs and Neutron Stars: The Physics of Compact Objects (Wiley, New York, 1983)

57. F. Sandin, P. Ciarcelluti, Astropart. Phys. 32, 278 (2009)

58. B. Kämpfer, J. Phys. A 14, L471 (1981)

59. B. Kämpfer, J. Phys. G 9, 1487 (1983)

60. J. Macher, J. Schaffner-Beilich, Eur. J. Phys. 26, 341 (2005)

61. B.K. Sharma, P.K. Panda, S.K. Patra, Phys. Rev. C 75, 035808 (2007)

62. J.L. Zdunik, P. Haensel, A\&A 551, A61 (2013) 